\title{
Multi-Temporal Speckle Filtering of Polarimetric P-Band SAR Data over Dense Tropical Forests: Study Case in French Guiana for the BIOMASS Mission
}

\author{
Colette Gelas ${ }^{1,2,3, *}$, Ludovic Villard ${ }^{1}$, Laurent Ferro-Famil ${ }^{1,4}$, Laurent Polidori ${ }^{1}$, Thierry Koleck ${ }^{1,2}$ \\ and Sandrine Daniel ${ }^{3}$
}

1 Centre d'Etudes Spatiales de la BIOsphère (CESBIO), Université de Toulouse, 31400 Toulouse, France; ludovic.villard@univ-tlse3.fr (L.V.); Laurent.Ferro-Famil@univ-rennes1.fr (L.F.-F.); laurent.polidori@ird.fr (L.P.); Thierry.Koleck@cnes.fr (T.K.)

2 Centre National d'Etude Spatial (CNES), 31400 Toulouse, France

3 Capgemini, 31100 Toulouse, France; sandrine.daniel@capgemini.com

4 Institut d'Électronique et des Technologies du numéRique (IETR), Université de Rennes 1, 35042 Rennes CEDEX, France

* Correspondence: glc.gelas@gmail.com

Citation: Gelas, C.; Villard, L.;

Ferro-Famil, L.; Koleck, T.; Daniel, S.; Polidori, L. Multi-Temporal Speckle Filtering of Polarimetric P-Band SAR Data over Dense Tropical Forests: Study Case in French Guiana for the BIOMASS Mission. Remote Sens. 2021, 13, 142. https://doi.org/10.3390/ rs13010142

Received: 20 October 2020 Accepted: 28 December 2020

Published: 4 January 2021

Publisher's Note: MDPI stays neutral with regard to jurisdictional claims in published maps and institutional affiliations.

Copyright: (c) 2021 by the authors. Licensee MDPI, Basel, Switzerland. This article is an open access article distributed under the terms and conditions of the Creative Commons Attribution (CC BY) license (https: / / creativecommons.org/ licenses/by/4.0/).

\begin{abstract}
The purpose of this paper is twofold, considering first the generalization of a multichannel speckle filter in order to handle temporal stacks of polarimetric SLC SAR data, and secondly the development of an ad hoc performance indicator based on the Polarimetric Orientation Angle (POA) in order to better estimate the resulting speckle reduction than the standard Equivalent Number of Looks (ENL) over densely vegetated regions, like tropical forests. Being based on the ability of PolSAR measurements to retrieve ground slopes through dense vegetation, this performance indicator requires the use of low frequencies such as P-band, as well as fully polarimetric data. This study has thereby a particular interest in the context of the upcoming BIOMASS spaceborne mission whose launch is scheduled in 2023, and makes use of data from the TropiSAR airborne campaign initiated in the early stage of the mission developments. Conducted over several test sites of tropical dense forests in French Guiana, this campaign gives us the opportunity herein to exploit P-band temporal stacks with repeated time intervals transposable to BIOMASS in terms of signal decorrelation. The application of the generalized multichannel speckle filter to the Paracou test site dataset reveals the limitations of the standard ENL analytical formula to assess speckle reduction in the case of spatially correlated media like dense forests, and for this purpose the interest of the correlation between POA and azimuthal slopes computed from an independent Digital Surface Model.
\end{abstract}

Keywords: multi-channel filtering (MCF); speckle filtering; equivalent number of looks (ENL); PolSAR; BIOMASS mission; polarimetric orientation angle

\section{Introduction}

P-band synthetic aperture radar is an imaging technique operating in the low frequency domain of micro-wave remote sensing, with frequencies ranging from $300 \mathrm{MHz}$ up to $1 \mathrm{GHz}$ (corresponding to wavelengths of $30 \mathrm{~cm}$ to $1 \mathrm{~m}$ ). Such waves can penetrate through dense media such as forested areas [1] and interact with scatterers large enough to infer the whole forest biomass. Given the core importance of forest Above Ground Biomass (AGB) for environmental issues including climate change (Forest AGB is part of Essential Climate Variable recognized by the United Nations Framework Convention on Climate Changes [2]), the unique sensitivity of P-band to forest AGB has pushed the development of BIOMASS as the seventh Earth Explorer mission by European Space Agency (ESA), to be launched in 2023. This mission will be the first $\mathrm{P}$ band space mission in repeat pass configuration, based on interval of about 3 days between acquisitions, whether for 
the so-called tomographic phase (with 7 passes) or the interferometric phase ( 3 passes). Indeed, the first 15 months of the mission will be dedicated to SAR tomography (TomoSAR) acquisitions and the rest of the mission to polarimetric SAR interferometry (PolInSAR). For such repeat-pass configuration, a key interest of P-band lies in its ability to maintain the coherence between image pairs to a sufficient level after several days (cf. [3]), hence this value of 3-days also constrained by orbit and coverage requirements [4]. This makes P-band much suitable than higher frequency bands to achieve repeat-pass TomoSAR and PolInSAR measurements from space. It can be also recalled that such measurements are particularly relevant at P-band since its penetration capabilities enable to retrieve signal contribution from all vegetation layers, until the ground one for most cases [1]. Besides, such penetration capabilities open the way to PolSAR based methods dedicated to the characterization of ground slopes, as proposed in [5] with the Polarimetric Orientation Angle (POA).

Although innovative, these data also rise questions about their behaviour regarding speckle effects, which are intrinsically related to the coherent sum of numerous contributions occurring within a resolution cell. A state-of-the-art review of the various speckle filters developed over the past few decades is provided in [6]. This paper deals with the development of an optimized filter adapted to PolSAR SLC (Single Look Complex) time series, to be further considered for the future BIOMASS data. Our purpose is then to assess the filter performances on SAR data over tropical forests (which concentrate the major scientific objectives of the mission), using the commonly used boxcar filter as reference. In order to assess their respective performances, the ENL (Equivalent Number of Looks) will be employed together with a new indicator based on the correlation between the POA and azimuthal slopes.

The paper is organized as follows. The data used for the study are described in Section 2. Section 3 presents the different steps of the filtering methods and the performance assessment is introduced. Results obtained with data acquired during the TropiSAR campaign in Amazonia (French Guiana, [7]) are presented in Section 4. The consistency and possible improvements of the filtering method are then discussed in Sections 5 and 6.

\section{Data Selection}

The study presented in this article is based on data acquired during TropiSAR campaign [7] over the Paracou test site (French Guiana), which offers an unique database particularly relevant to simulate the future PolSAR time series of BIOMASS data. Indeed, a sequence of 7 PolSAR images have been acquired in about one month, with variable time intervals from 2 to 7 days. These time intervals are very close in term of temporal decorrelation to the 3 days planned for BIOMASS, as shown by previous studies [8] based on the TropiScat experiment (from 2011 to 2014, cf. [3]), also part of the Paracou test site and representative of tropical dense forest. Moreover, this dataset includes not only the original full bandwidth version (125 MHz, meaning a range resolution about $1 \mathrm{~m})$, but also a reprocessed version at $6 \mathrm{MHz}$ to simulate the future spaceborne configuration of BIOMASS.

Based on previous work on this study case [9], we also use the DEM (Digital Elevation Model) from SRTM products in order to characterize the effects of terrain slopes. Since the SRTM DEM is derived from short wavelength radar data (C-band, i.e a wavelength of $6 \mathrm{~cm}$ ), it is important to recall that this product only provides elevations at canopy level, while it is used to account for the topographic effects at ground level. In order to overcome this limitation, we use the $90 \mathrm{~m}$ resolution product which is supposed to be less impacted by forest gaps or forest/non-forest transitions than the $30 \mathrm{~m}$ product. Indeed, slope errors caused by the use of canopy elevations to estimate ground slopes can be significantly reduced by a degradation of the DEM resolution, as shown in [10] for similar tropical forest areas in Brazil.

It is also worth to note that the Paracou test site is characterized by a variable terrain topography, from flat areas to rather hilly ones with significant effects on the SAR images [9]. This region is also mostly covered by typical tropical rainforest with a very high density 
and diversity of trees, but also includes bare soil areas, a river and human settlements. As detailed in [11], this test site can also benefit from high quality in-situ measurements, distributed over several representative regions of interest (ROI) covering about 84 ha. Depending on purpose, these ROI can be used at several scales (from $25 \times 25 \mathrm{~m}^{2}$ to $25 \mathrm{ha}$ ). In order to get a suitable trade-off between AGB estimates [12], geolocation and overlapping errors, we use subplots from 6.25 ha to 1 ha subplots as detailed in [11], and as shown in the result section hereinafter.

\section{Multi Temporal and Multi Channel Speckle Filter}

The proposed Multi Temporal and Multi Channel filter is an extension of the scalar multi-temporal approach proposed in [13] to the case of multivariate data, i.e., acquired with an arbitrary mode of diversity, here over different polarization channels.

\subsection{Intensity-Based Multi-Temporal Filtering Techniques}

The objective of a multi temporal speckle filter is to estimate $\sigma=E(\mathbf{p})$, the expectation of $\mathbf{p}=\left[I_{1}, \ldots, I_{k}\right]^{T} \in \mathbb{R}^{+K}$, a multi-temporal vector composed of $k$ multi-looked intensity values sampled at different dates. Among the different possible approaches, the solution retained in [13] proposes to perform this estimation using a linear filter

$$
\mathbf{r}=\mathbf{W}^{T} \mathbf{p} \quad \text { with } \quad \mathbf{W}=\left[\mathbf{w}_{1}, \ldots, \mathbf{w}_{K}\right] \in \mathbb{R}^{K \times K}
$$

where $\mathbf{w}_{k}$ represents the $K$-element filter used to compute $r_{k}$, the estimate of $\sigma_{k}$, by linearly combining the different elements of $\mathbf{p}$. The quality of a speckle filter is often measured through the equivalent number of looks, ENL, of the filtered intensity [14], defined under the assumption of a fully developed speckle (for normally distributed complex data) [15] as

$$
L_{r_{k}}=\frac{E^{2}\left(r_{k}\right)}{\operatorname{var}\left(r_{k}\right)}
$$

Maximizing the output ENL under the constraint of mean preservation, that is $E\left(r_{k}\right)=$ $\sigma_{k}$, leads to the following linearly constrained minimum variance optimization problem

$$
\widehat{\mathbf{w}}_{k}=\underset{\mathbf{w}_{k}}{\arg \min } \operatorname{var}\left(r_{k}\right) \quad \text { subject to } \quad E\left(r_{k}\right)=\mathbf{w}_{k}^{T} \sigma=\sigma_{k}
$$

whose solution writes

$$
r_{k}=f \sigma_{k} \quad \text { with } \quad f=\frac{\sigma^{T} \mathbf{C}^{-1} \mathbf{p}}{\boldsymbol{\sigma}^{T} \mathbf{C}^{-1} \boldsymbol{\sigma}} \quad \text { and } \quad \mathbf{C}=\mathrm{E}\left((\mathbf{p}-\sigma)(\mathbf{p}-\sigma)^{T}\right)
$$

where $f$ is a filtering coefficient which does not depend on the filtered channel, $\mathbf{C}$ the covariance matrix of the input intensities and $E(\mathbf{r})=\sigma$ [13]. The expression in (4) contains unknown second-order quantities, $\sigma$, and a fourth-order matrix, $\mathrm{C}$, whose accurate estimation generally requires numerous samples.

As shown in [14], the equivalent number of looks after filtering is then given, according to (2), by

$$
L_{e q}=\sigma^{T} \mathbf{C}^{-1} \sigma
$$

Under the assumption of uncorrelated multi-temporal intensities, $\mathrm{C}$ is diagonal and the different components of the filtering coefficient become

$$
L_{e q}=\sigma^{T} \mathbf{C}^{-1} \sigma=\sum_{k=1}^{K} \frac{\sigma_{k}^{2}}{\operatorname{var}\left(I_{k}\right)}=\sum_{k=1}^{K} L_{I_{k}}
$$


with $L_{I_{k}}$ the ENL of $I_{k}$. Likewise with C diagonal it follows that :

$$
\sigma^{T} \mathbf{C}^{-1} \mathbf{p}=\sum_{k=1}^{K} \frac{\sigma_{k}^{2}}{\operatorname{var}\left(I_{k}\right)} \frac{I_{k}}{\sigma_{k}}=\sum_{k=1}^{K} L_{I_{k}} \frac{I_{k}}{\sigma_{k}}
$$

which makes possible to define the filtering coefficient $f$ as :

$$
f=\frac{1}{L_{e q}} \sum_{k=1}^{K} L_{I_{k}} \frac{I_{k}}{\sigma_{k}}
$$

At this stage, it is worth to note that this formulation considers the regional estimates $\sigma_{k}$ for the intensities as priors to the higher resolution ones $r_{k}$, under the hypothesis of spatial stationarity whose validity is likely to be effective for small regions, hence the semi-empirical choices further detailed to optimize this trade-off.

\subsection{Extension to SLC Data}

\subsubsection{General Form}

In the multi channel case, $K$ coherent vector acquisitions performed at different dates, $\left\{\mathbf{k}_{k}\right\}_{k=1}^{K}$, are used to compute second-order matrix representations, $\left\{\mathbf{T}_{p_{k}}\right\}_{k=1}^{K}, \mathbf{T}_{p_{k}}$ being an estimate of the $(N \times N)$ covariance matrix of $\mathbf{k}_{k}$. Replacing $I_{k}$ by $\mathbf{T}_{p_{k}}$ in (1) and (4) leads to the following expression for the MCMT filter :

$$
\mathbf{T}_{r_{k}}=f_{M C} \mathbf{T}_{\sigma_{k}}
$$

where $f_{M C}$ represents a filtering coefficient that is common to all the acquisition dates, as in the scalar case, and to all the channels, though preserving any distortion of the multivariate information during the filtering process. In order to account for the statistical behavior of all the processed channels and date, the filtering coefficient is computed as in (4), using vectors containing multi temporal and multi channel intensities

$$
\mathbf{x}_{M C}=\operatorname{vec}\left(\operatorname{diag}\left(\mathbf{T}_{x_{1}}\right), \ldots, \operatorname{diag}\left(\mathbf{T}_{x_{k}}\right)\right) \in \mathbb{R}^{+N K} \quad \text { with } \quad \mathbf{x}=\mathbf{p}, \sigma
$$

where $\operatorname{diag}(\mathbf{X})$ provides a vector containing the elements located on the diagonal of $\mathbf{X}$. The expressions of $f_{M C}$ may be obtained from (4) and (8) by replacing $\mathbf{p}$ and $\sigma$ by their multi channel and date counterparts, $\mathbf{p}_{M C}$ and $\sigma_{M C}$, respectively, defined in (10). A synopsis of the MCMT filter is given in Figure 1 where the red rectangular blocks represent input datasets and blue blocks with rounded corners represent the processing steps.

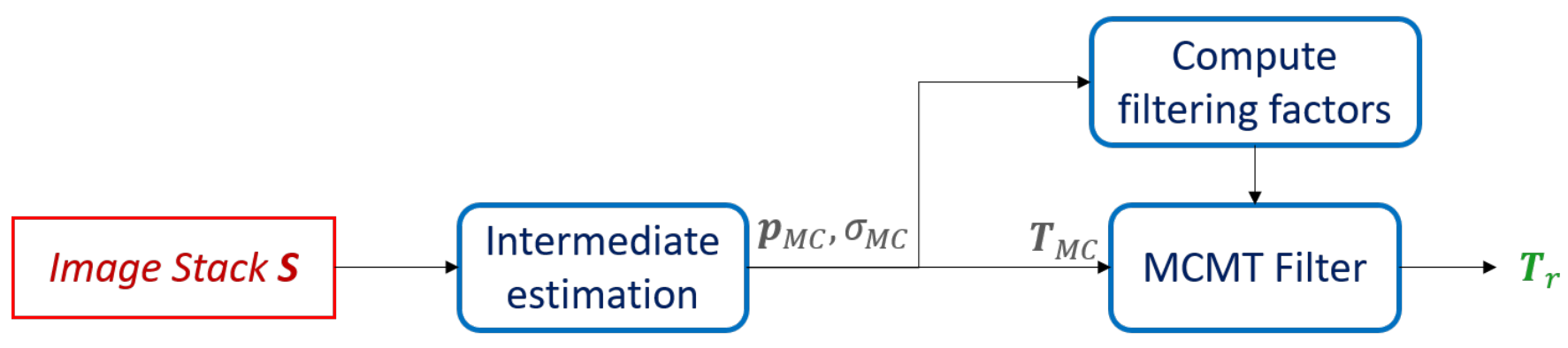

Figure 1. Synopsis of the proposed MCMT filter.

\subsubsection{Application to Polarimetric SAR Data}

Fully polarimetric coherent acquisitions may be represented using a three-element target vector, $\mathbf{k}$, from which one may compute a polarimetric coherency matrix as

$$
\mathbf{T}=\mathrm{E}\left(\mathbf{k k}^{* T}\right) \quad \text { with } \quad \mathbf{k}=\frac{1}{\sqrt{2}}\left[\begin{array}{lll}
S_{H H}+S_{V V}, & S_{H H}-S_{V V}, & \sqrt{2} S_{H V}
\end{array}\right]^{T}
$$


One may show that, due to the semi definite positiveness of the second-order representation in (11), the filtering coefficient in (9) is a positive real number. In the case of a multi-temporal acquisition performed over a period of time small enough so that the $K$ measurements depict a stationary behavior, one may compute a mean estimate using an additional boxcar filter operating in the time direction.

$$
\mathbf{T}_{r}=\frac{1}{K} \sum_{k=1}^{K} \mathbf{T}_{r_{k}}
$$

\section{Results}

In this section, the contributions of the MCMT filter on the time series of the Paracou test site presented in the Section 2 have been studied. The reference filter used to estimate the contribution of the MCMT filter is a widely used boxcar filter, noted REF, separately applied to each image of the stack, i.e., which does not take into account the temporal dimension. Three criteria were used to evaluate the properties and performance of the MCMT filter. The first criterion, relating to the filter properties, is to evaluate the variations of the average filter intensity through the $t^{0}$ indicator presented in [9]. The next two criteria are used to estimate the filter performances through the ENL and the azimuthal slopes estimated from the filtered P-band data. The last indicator, related to azimuthal slopes, is specific to the use of PolSAR P-band data which are particularly sensitive to topographic effects.

\subsection{Implementation}

We considered full resolution data obtained with $125 \mathrm{MHz}$ bandwidth. The interest of this time series is that it perfectly respects the BIOMASS acquisition protocol, with an acquisition interval time of several days that allows us to observe an intensity correlation corresponding to a moment of order 4 that oscillates around 0.6 equivalent to a coherence about 0.8 [3].

Despite the high correlation values between the different components of the time series, we chose to apply the version of the MCMT filter that does not take the correlation into account. Indeed, calculating a moment of order 4 for a time series of this length is very time consuming because the number of samples required for an accurate estimate of $\mathbf{C}$ is very large. The choice of this sub-optimal but more robust version of the MCMT filter is discussed in Section 5.

As detailed in (11) we calculate the coherence matrices $\mathbf{T}$ for each component of the time series by applying a boxcar filter to perform a spatial smoothing of the speckle. We can thus define $\mathbf{p}_{M C}=\operatorname{vec}\left(\mathbf{T}_{x_{1}}, \ldots, \mathbf{T}_{x_{k}}\right)$ to estimate the contributions of the MCMT filter. We also define the $\sigma_{M C}=\operatorname{vec}\left(\operatorname{diag}\left(\mathbf{T}_{x_{1}}\right), \ldots, \operatorname{diag}\left(\mathbf{T}_{x_{k}}\right)\right)$ where the filter window applied to $\sigma_{M C}$ is twice as large as that applied to $\mathbf{p}_{M C}$, respecting local homogeneity constraints. The MCMT filtering is then applied following the steps of Figure 1. The time series data are also filtered with the REF filter which corresponds to a boxcar with a window size equivalent to $\sigma_{M C}$ to get a reference.

Figure 2 allows to observe in slant range resolution the contribution of MCMT filtering compared to REF for one of the components $T_{i j}^{k}$ from $\mathbf{p}_{M C_{k}}$. The two maps on the left are obtained after applying the REF and MCMT filters on the Paracou time series for an output resolution equivalent to $5 \mathrm{~m}$. The two maps on the right have a resolution of $50 \mathrm{~m}$ equivalent to the future resolution of the BIOMASS mission data. The differences between the images filtered with REF or MCMT are not visually significant, so that we prefer a quantitative assessment in the following analysis. For this study, four regions of interest (ROI), visible on the maps to the left of the Figure 2, were established on areas of forest or savannah chosen as homogeneous as possible. Within the framework of the BIOMASS mission, field measurements are also available in the same area as ROI 1 [11]. 
(a) Paracou $125 \mathrm{MHz}$ [ Multilook $5 \mathrm{~m}$ ]

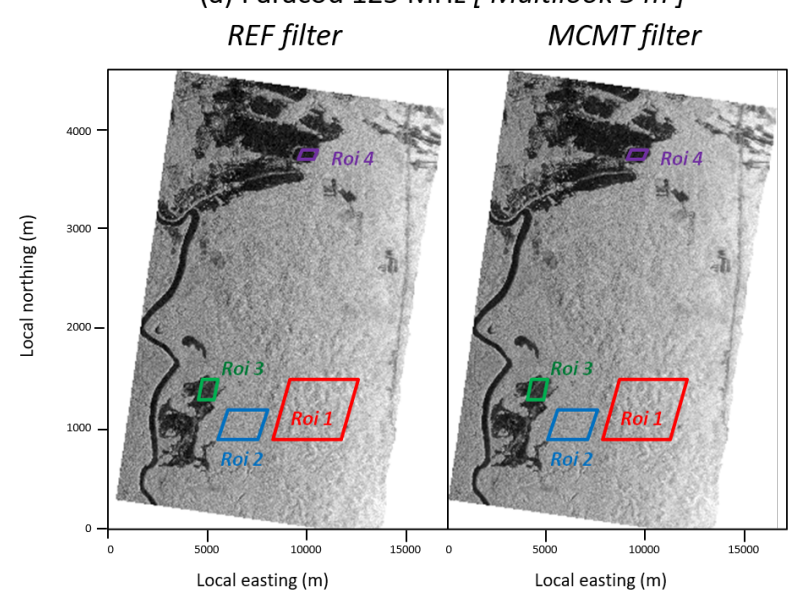

(b) Paracou $6 \mathrm{MHz}$ [ Multilook $50 \mathrm{~m}$ ]

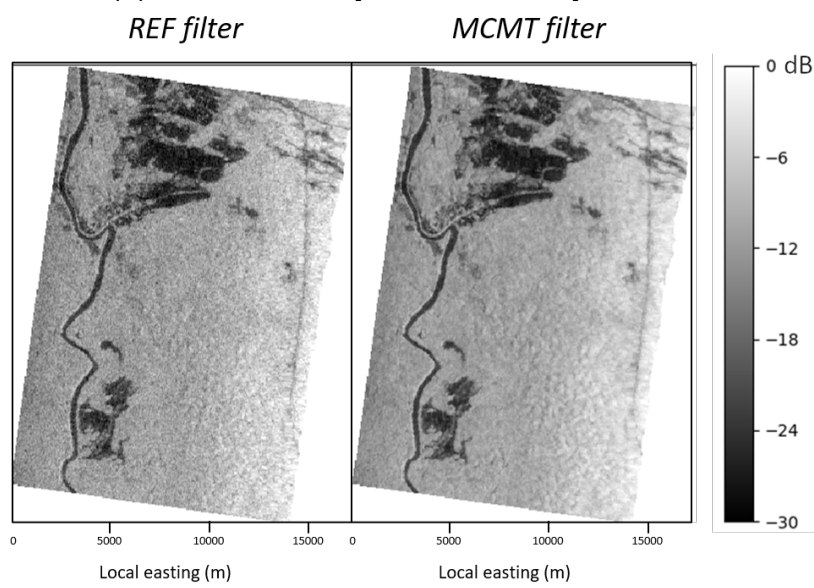

Figure 2. Results of REF and MCMT filtering of an image $T_{33}=4\left\langle S_{H V}^{*}\right\rangle$ from the data from the Paracou test site acquired during the TropiSAR airborne campaign. The two maps on the left have an output resolution of $5 \mathrm{~m}$, the two maps on the right have an output resolution of $50 \mathrm{~m}$.

\subsection{Preservation of the Average}

Overall, speckle filters do not necessarily preserve the average values of the filtered intensities. For our concerns in the frame of forest AGB retrieval, average preservation is essential, as pointed out by the calibration constrains on the future BIOMASS mission [16,17], or by the developments of backscattering coefficients to account from geometrical and scattering effects which may impact the raw intensities.

In order to minimise these effects, PolSAR P-band intensities are commonly studied through polarimetric indicators, expressed as a function of forest biomass, which allow to take into account the data acquisition geometry and the disturbing effects related to forest volume or local topography [7]. The polarimetric indicator $t^{0}$, detailed in [9], uses all the polarisations of the intensity matrix $\mathbf{S}$, transposed into the coherence matrix $\mathbf{T}$, to minimise disturbance effects. This feature makes $t^{0}$ a better indicator to quantify the properties of the MCMT filter in terms of conservation of the average intensity and highlights the importance of independently filtering all the polarimetric and temporal channels of $\mathbf{T}$ to maximise the $t^{0}$ performances.

Its expression is recalled in (13), where $N^{0}$ corresponds to the normalization coefficient related to the minimization of geometric effects and double bounce phenomena, and $T_{33}^{\Theta}$ is the coefficient $T_{33}$ of $\mathbf{T}$ after application of the $\Theta$ rotation related to the polarimetric orientation angle that minimizes the effects of local topography [9].

$$
t^{0}=\left\langle\frac{1}{N^{0}} \cdot T_{33}^{\Theta}\right\rangle
$$

Figure 3 (left side), shows the $t^{0}$ values as a function of forest biomass from different ROIs presented in the Section 2 which range from 6.25 to 25 ha. On Figure 3 (right side), the ROIs have been divided into 1 ha plots, thus considerably increasing the number of available ROIs. The results from the REF filtering are shown in red, those from the MCMT filtering are shown in blue. For each type of filtering, a first logarithmic model has been estimated from the ROI and their parameters are detailed in the Table 1 below. 

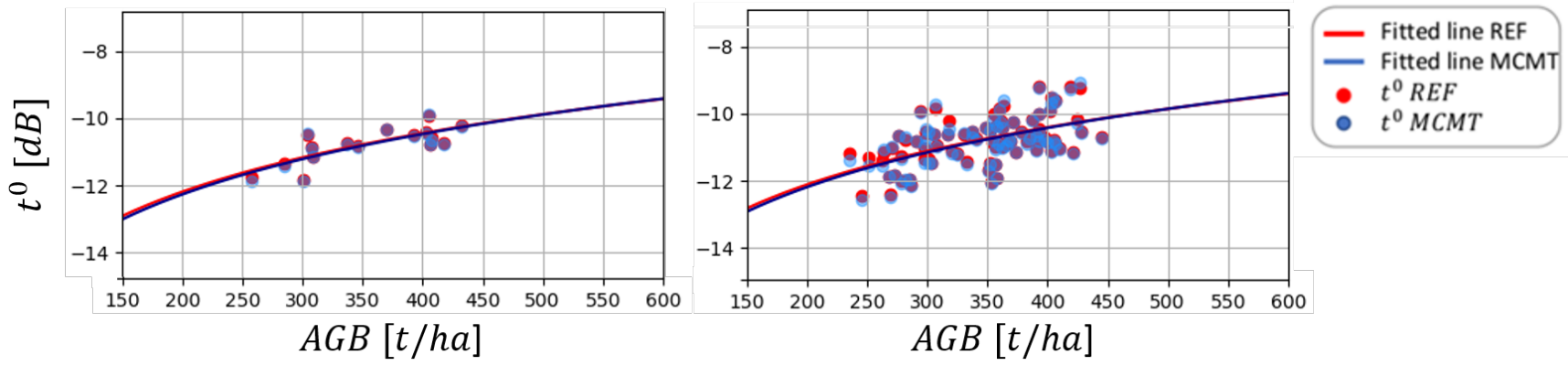

Figure 3. Estimation of $t^{0}$ as a fonction of forest biomass obtained for the P-band PolSAR data from the Paracou test site acquired with a bandwidth of $125 \mathrm{MHz}$ with a resolution after filtering equivalent to $5 \mathrm{~m}$. On the left, 15 ROIs of 6.25 ha are considered as well as 1 ROI of 25 ha. On the right, 84 ROIs of 1 ha are considered.

Table 1. Parameters of regression models estimated for $t^{0}=f(A G B)$ shown in Figure 3 and associated statistics (Pearson Coefficient $\left(r_{p}\right)$, Root Mean Squared Error (RMSE), $\chi^{2}$ parameter).

\begin{tabular}{cccccc}
\hline 16 ROIs & $\mathrm{a}$ & $\mathrm{b}$ & $r_{p}$ & RMSE & $\chi^{2}$ \\
\hline REF & 5.79 & -25.51 & 0.78 & 21.84 & 5.13 \\
MCMT & 5.97 & -26.0 & 0.76 & 19.37 & 5.33 \\
\hline 84 ROIs & $\mathrm{a}$ & $\mathrm{b}$ & $r_{p}$ & RMSE & $\chi^{2}$ \\
\hline REF & 5.65 & -25.12 & 0.54 & 33.63 & 27.14 \\
MCMT & 5.84 & -25.62 & 0.54 & 33.84 & 32.53 \\
\hline
\end{tabular}

Figure 3 and Table 1 show the very strong similarity between the regression models estimated after application of the REF and MCMT filters on T, confirming that the MCMT filter allows the conservation of the mean intensities allowing a gain in resolution compared to the REF filter. To illustrate this property, Figure 4 shows the values of $t_{M C M T}^{0}$ as a function of $t_{R E F}^{0}$ for the two cases shown in Figure 3. In both cases, the data are perfectly aligned on the 1:1 reference line, which means that the MCMT filter retains the average intensities.
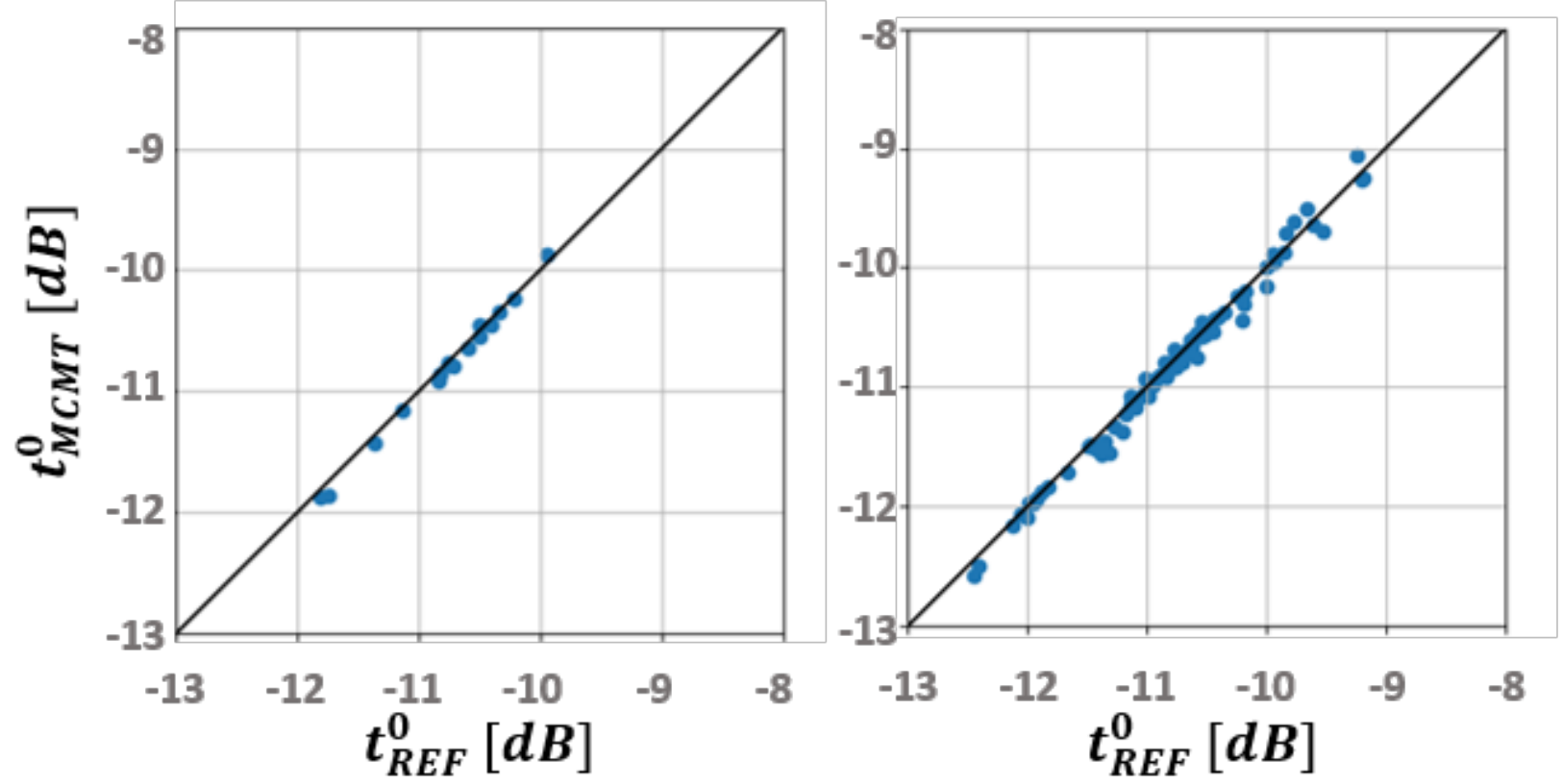

Figure 4. Estimation of $t_{M C M T}^{0}$ as a function of $t_{R E F}^{0}$ obtained for the P-band PolSAR data from the Paracou test site acquired with a bandwidth of $125 \mathrm{MHz}$ with a resolution after filtering equivalent to $5 \mathrm{~m}$. On the left, $15 \mathrm{ROIs}$ of 6.25 ha are considered as well as 1 ROI of 25 ha. On the right, 84 ROIs of 1 ha are considered. 


\subsection{Analysis in Terms of Speckle Reduction}

\subsubsection{The Equivalent Number of Looks (ENL)}

The study of ENL improvement is anchored in the process of quantifying the performance of a speckle filter. In this paper, the contribution of the MCMT filter in terms of ENL improvement has been studied as a function of the output data resolution after filtering.

Four ROIs have been defined in Figure 2 to study the variation in ENL between data filtered simply with REF and data that have been filtered with the MCMT filter. The ENL, noted $L_{e q}$ and defined in (2) is represented in Figure 5 depending on the resolution of the data obtained after filtering. Figure 5 (left side) shows that for the 4 targeted ROIs, the ENL after MCMT filtering is greater than using simply REF filtering. The trend curves confirm the contribution of the MCMT filter on the $L_{e q}$. However, we note that the dispersion of the $L_{e q}$ is increasing between the ROIs when the resolution becomes coarser.
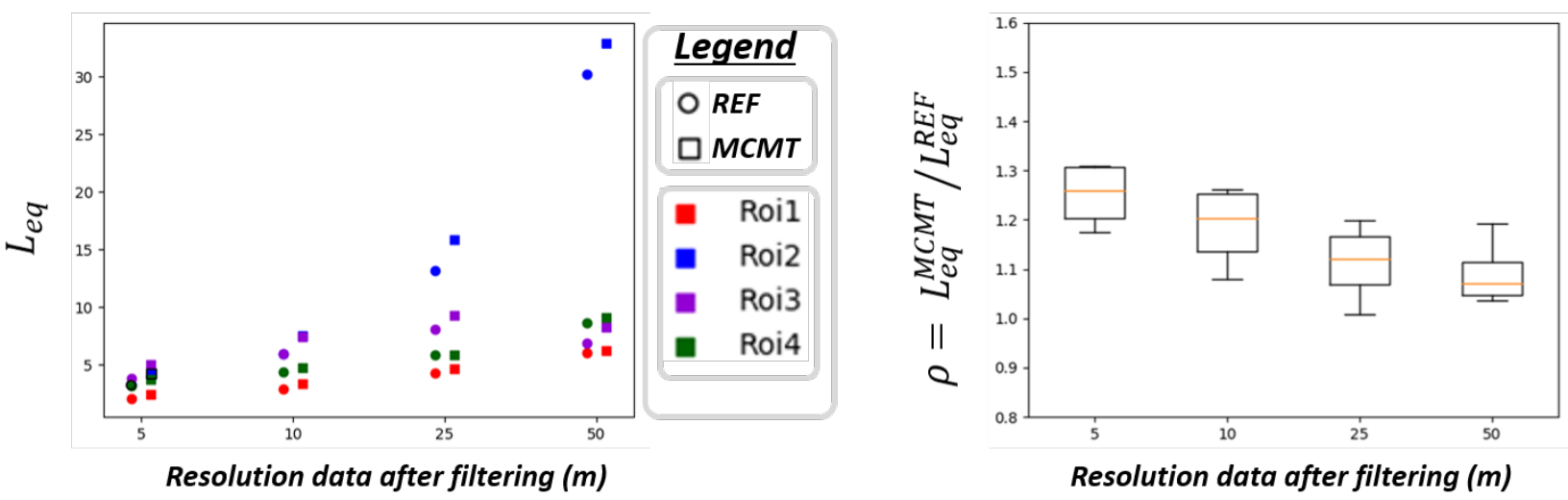

Figure 5. Impact of the multilook pixel size on the equivalent number of looks $L_{e q}(\mathbf{l e f t})$ and on the ratio $\rho=L_{e q}^{M C M T} / L_{e q}^{R E F}$ (right) for the 4 ROIs shown in Figure 2.

In general, speckle filtering allows a clear increase in ENL according to the filtering spatial resolution through a progressive smoothing of the speckle. In the study presented here, regardless of the filter used, the growth of ENL is very limited, as shown in Figure 5 (left side). This phenomenon is expected under the common statistical assumptions (independent and identically distributed realizations between each pixel).

To illustrate the added-value of the MCMT in comparison to the REF filter, the evolution of the ratio $\rho=L_{e q}^{M C M T} / L_{e q}^{R E F}$ can be seen in Figure 5 (right side). The ratio $\rho$ is always greater than 1 which confirms that the $L_{e q}$ from MCMT filtered data is always higher than those obtained with REF filtered data. We also note that even if the standard deviation of the ratios around the mean remains rather constant, the added-value of the MCMT tends to decrease. This phenomenon can be explained by the homogeneity hypothesis which becomes questionable for such large spatial multilook, and also by the higher temporal correlation between larger regions from the image pairs.

Actually, this result illustrates very well the impact of pixel correlation within specific regions of the image, especially between ROIs 1 and 2: both are covered by a dense vegetation but ROI 1 is characterized by a significant terrain topography which generates a higher level of inter-correlation between pixels, hence a degraded efficiency of the multilook (and smaller ENL). This inter-correlation between pixels implies violation of the independent and identically distributed pixels hypothesis, which makes hazardous the prediction of ENL as a function of filtering spatial resolution. As a result, the use of the Gaussian based hypothesis analytical Formula (2) for the ENL is not necessary reliable in all cases, and particularly not for forested areas under hilly terrains. To overcome this limitation, the quality of the aforementioned POA estimate can investigated, as proposed in the next subsection. 


\subsubsection{Estimation of the Polarimetric Orientation Angle $\left(\hat{\psi}_{N}^{a z}\right)$}

The unique sensitivity of P-band microwaves to forest AGB is related to their penetration capabilities, which in addition open the way to the characterization of the underlying terrain topography not only from interferometric or tomographic measurements but also from PolSAR data. Indeed, it has been shown in [5] that azimuthal slopes can be estimated from a rotation around the line of sight (LoS) of the coherency matrix $\widehat{T}$, although the questions about the perturbing effects of dense vegetation on this method remain open. This slope estimation results from the polarisation basis orientation which maximizes the co-polarization response. The method is recalled in (14) where $T_{22}, T_{23}, T_{33}$ are the terms of the $\widehat{\mathbf{T}}$ matrix described in (11).

$$
\widehat{\psi}_{N}^{a z}=\left[\tan ^{-1}\left(\frac{-4 \operatorname{Re}\left(\mathbf{T}_{23}\right)}{-\mathbf{T}_{22}+\mathbf{T}_{33}}\right)+\pi\right] / 4
$$

If $\widehat{\psi}_{N}^{a z}>\pi / 4$, it is replaced by $\left(\widehat{\psi}_{N}^{a z}-\pi / 2\right)$. The results of estimated $\widehat{\psi}_{N}^{a z}$ as a function of the multilook pixel size for full resolution acquisitions are shown in Figure 6. The image on the far left of the figure corresponds to the azimuthal slopes $\psi_{N}^{A z}$ extracted from the SRTM DEM using the method described in [9]. The four images, arranged in two lines on the right side of the Figure 6, correspond to the $\widehat{\psi}_{N}^{a z}$ estimated from the filtered coherency matrices $\widehat{\mathbf{T}}_{R E F}$ and $\widehat{\mathbf{T}}_{M C M T}$ with a spatial resolution at the output of the filter corresponding to $25 \mathrm{~m}$ and $50 \mathrm{~m}$. Figure 7 is a zoom of the area framed in red on Figure 6, organised in the same layout which targets an area of variable topography that was chosen for its very hilly topography with a high density of forest.

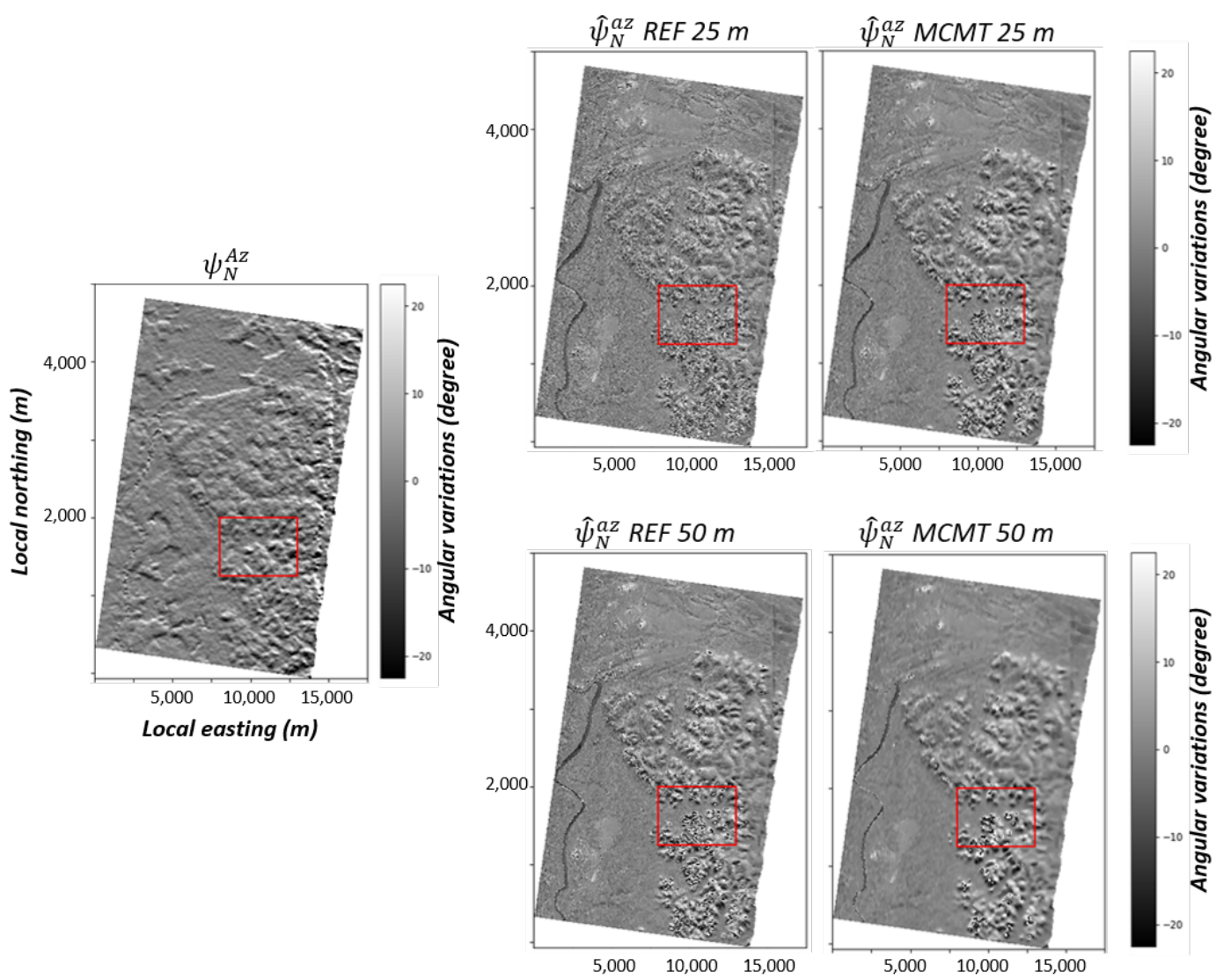

Figure 6. Azimuthal slopes estimation from Paracou test site. Image on the left side corresponds to azimuthal slopes $\psi_{N}^{A z}$ extracted from the SRTM DEM. The four images, arranged in two lines on the right side, corresponds to azimuthal slopes $\widehat{\psi}_{N}^{a z}$, estimated from filtered coherency matrices $\widehat{\mathbf{T}}_{R E F}$ and $\widehat{\mathbf{T}}_{M C M T}$ with a spatial resolution at the output of the filter corresponding to $25 \mathrm{~m}$ and $50 \mathrm{~m}$. The red box corresponds to the area of interest selected for Figures 7 and 8 . 

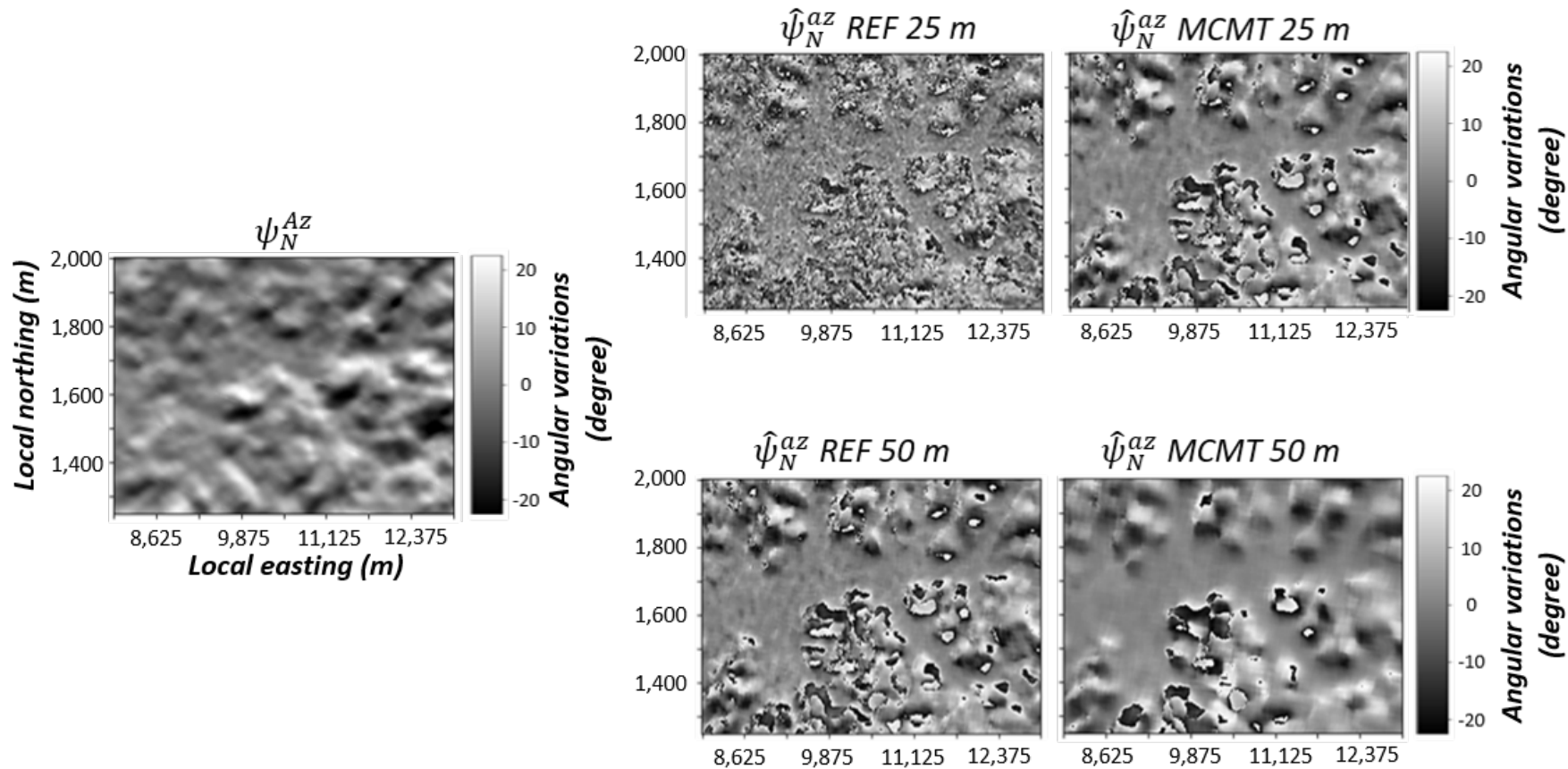

Figure 7. Zoom corresponding to the red rectangle in Figure 6. Image on the left side corresponds to azimuthal slopes $\psi_{N}^{A z}$ extracted from the SRTM DEM. The four images, arranged in two lines on the right side, corresponds to azimuthal slopes $\widehat{\psi}_{N}^{a z}$, estimated from filtered coherency matrices $\widehat{\mathbf{T}}_{R E F}$ and $\widehat{\mathbf{T}}_{M C M T}$ with a spatial resolution at the output of the filter corresponding to $25 \mathrm{~m}$ and $50 \mathrm{~m}$.

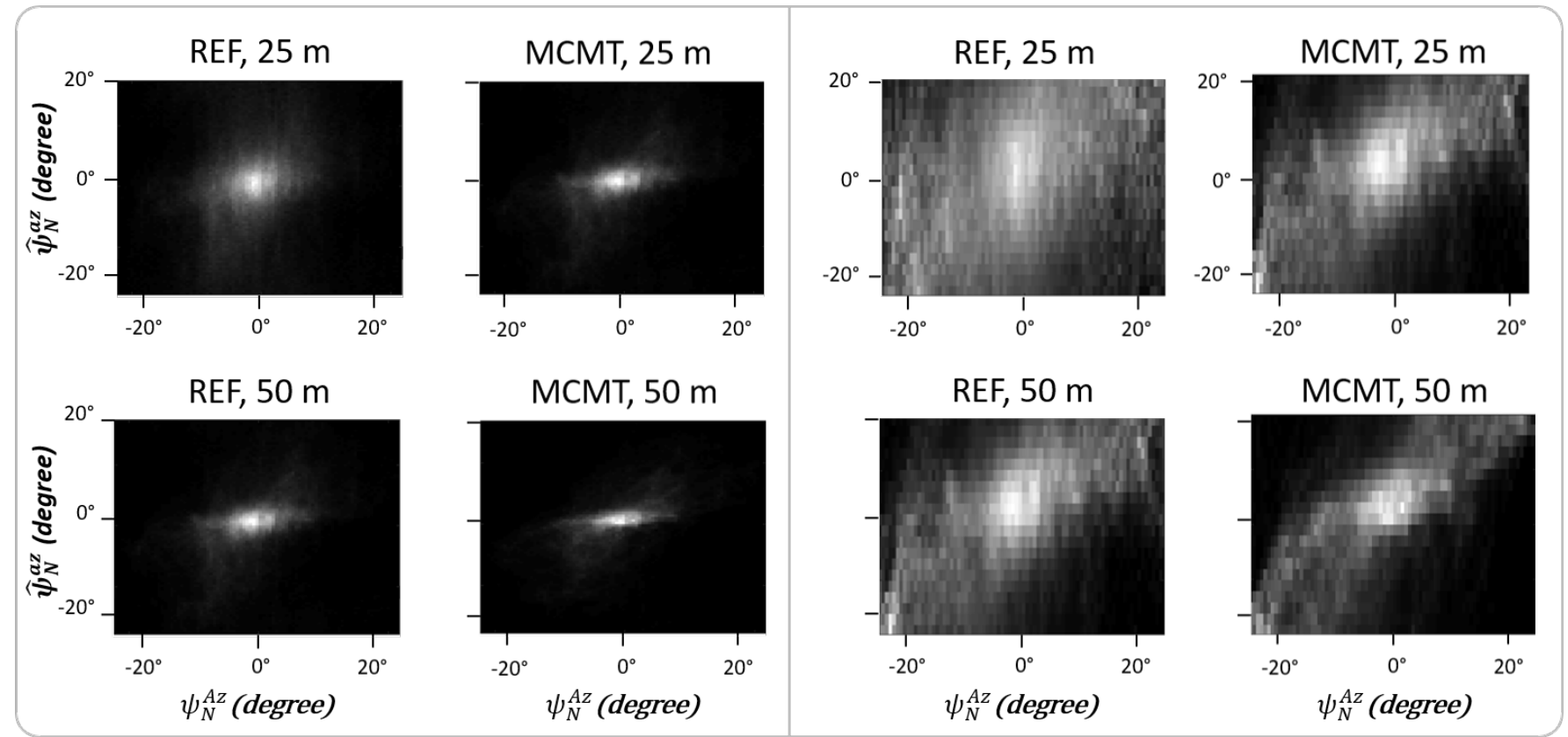

Figure 8. In the left block, 2D histograms of $\psi_{N}^{a z}$ versus $\widehat{\psi}_{N}^{a z}$ computed for the red box area of the Figure 6 . In the right block, 2D histogram of the left block with local slope filtering. On the first line, results obtained with a resolution of $25 \mathrm{~m}$ at the filter output, on the second line, results obtained with a resolution of $50 \mathrm{~m}$ at the filter output.

Figures 6 and 7, highlight the similarities between the azimuthal slopes of the SRTM DEM and the topography estimated from $\widehat{\psi}_{N}^{a z}$. They illustrate the impact of the spatial resolution of filtering on the estimation of azimuthal slopes. Areas of very changing topography remain very uncertain despite the increase in the spatial resolution of filtering. In the same way, the speckle that is visible for small spatial filtering resolutions (REF filter at $25 \mathrm{~m}$ ) completely disappears with large multilook pixel size. MCMT filtering results give 
more homogeneous maps, where remarkable regions are smoothed and identifiable for smaller resolution and the number of areas of uncertainty with rapid variations in slope is reduced compared to REF filtering results. On the zoom of Figure 7, details corresponding to noise are notably observable for the $\hat{\psi}_{N}^{A z}$ REF with the multi-look equivalent to $50 \mathrm{~m}$ resolution and not for the $\hat{\psi}_{N}^{A z}$ MCMT.

Using the reference azimuthal slopes $\psi_{N}^{A z}$ from the $30 \mathrm{~m}$ SRTM DEM visible on the left of Figure 6, it is possible to estimate the robustness of the estimated slopes from PolSAR time series. The 2D histograms observed on the left of Figure 8 shows the correlation of the $\widehat{\psi}_{N}^{a z}$ with $\psi_{N}^{A z}$ for several spatial resolution filtering on the reduced area framed in red on Figure 6. The 2D histograms on the right of the Figure 8 have been normalised based on the local slope, so that areas with flat topography are not taken into account in the expression of the correlation.

Figure 8 shows that the correlation between $\psi_{N}^{A z}$ and $\widehat{\psi}_{N}^{a z}$ increases proportionally with the spatial resolution filtering. This is explained by the decrease in rapid slope variations and the gradual disappearance of the speckle. The dispersion around the diagonal of the histogram shows that the data filtered with REF are much more noisy than those obtained with the MCMT filter. The comparison of MCMT and REF filtering shows that the results are equivalent to within one resolution. This confirms the impact of the MCMT filter on the texture with a reflectivity maintenance.

The study was also carried out for data at $6 \mathrm{MHz}$ for 50, 100 and $200 \mathrm{~m}$ multilook pixel. The results obtained at 50 and $100 \mathrm{~m}$ are very interesting with the MCMT filter with correlations between 0.2 and 0.3 as opposed to values below 0.2 for the REF.

This first study on the Paracou site allows to conclude that the radar information related to the slope is contaminated by speckle at the largest multilook pixel size. Speckle filtering allows to improve slope estimation at the pixel scale. However, it implies a degradation of the multilook pixel size. The MCMT filter improves the result by having less impact on the multilook pixel size. However, the contribution of the MCMT filter decreases as the resolution of the multi-look increases. The latter is advantageous when using data acquired with limited native resolution, where filtering the speckle while preserving the resolution is a priority, as in the case of the future BIOMASS mission where the bandwidth will be $6 \mathrm{MHz}$.

The study carried out in this paper showed the interest of using several indicators to quantify the contributions of a multi-channel and multi-temporal filter applied to PolSAR time series related to the observation of dense forests. The comparison of the results obtained using the REF and MCMT filters have shown that the POA is a more decisive performance indicator than the standard ENL and is truly decisive for the P-band PolSAR data specific to the future BIOMASS mission. To synthesised our approach, the process we used to compare the performance of the REF and MCMT filters is represented by the flowchart given in Figure 9. The red rectangular blocks represent input datasets and the blue blocks with rounded corners represent the processing steps. 


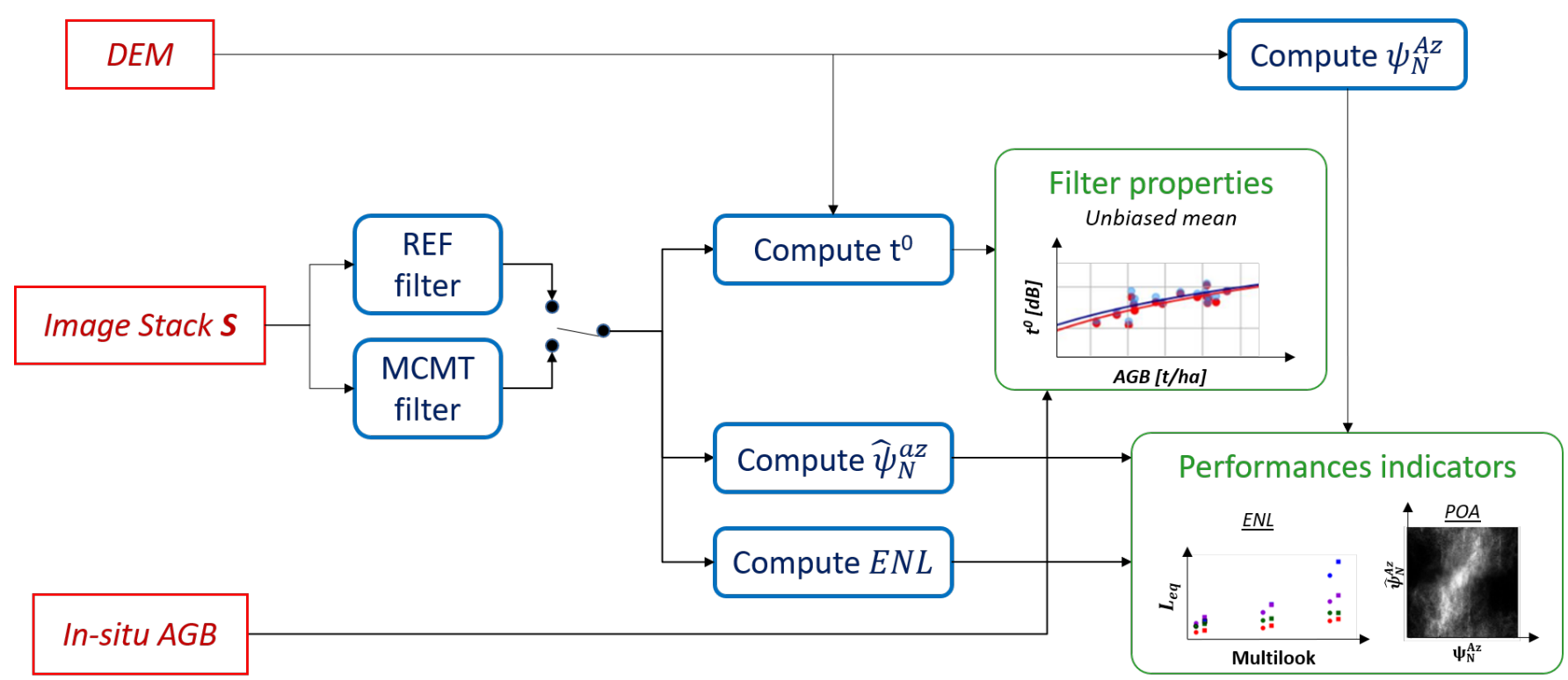

Figure 9. Flowchart representing the overall process for filtering P-band PolSAR time series and estimating properties and performances indicators developed in this paper.

\section{Discussion}

For this article, we used data from the Paracou test site in French Guiana. This site is known for the very dense structure of its forest cover as well as for its hilly topography. The acquisitions made there during the airborne campaign TropiSAR were made in accordance with the acquisition protocol of the future BIOMASS mission. A delay of 3 to 4 days was respected between each shot, taken at an ideal road angle to maximize backscatter phenomena related to ground contributions. As demonstrated in [9], the Paracou test site is very well adapted to topography estimation and to the use of the $t^{0}$ polarimetric indicator for biomass estimation.

We have chosen not to use the data acquired during the AfriSAR campaign [18] because of the acquisition protocol deployed. Indeed, the AfriSAR campaign was devoted to TomoSAR acquisitions as a priority, so that we do not have PolSAR time series exceeding 3 acquisition dates. Moreover, the time intervals between the different acquisitions of the same series are on the other side of minutes, to respect the TomoSAR configuration and keep a maximum of coherence between the data. Too much consistency between the data does not allow an optimal use of the MCMT filter because there are no differences exploitable enough for the calculation of the $f$ filtering term. These elements contribute to the exclusion of the AfriSAR campaign for this article.

The use of supposedly decorrelated data for the MCMT filter raises an important point regarding the quantification of the gain in terms of equivalent look number $L_{e q}$ if correlation data is used. For this quantification, we used the TropiScat data which, using a semi-empirical decorrelation model, allowed us to estimate the decorrelation of the data as a function of time as shown in Figure 10 (left side). Several levels of correlation were represented from non-diagonal $\mathbf{C}$ matrices. From the methods proposed in [13], it is possible to estimate the gain in $L_{e q}$ obtained after using a MCMT filter. Figure 10 (right side) shows the theoretical $L_{e q}$ obtained after filtering for 3 correlation levels $0.3,0.5$ and 0.7 as a function of time. The solid lines are for MCMT filtering which takes into account the decorrelation of the data. The dashed lines are for the MCMT filtering proposed in this paper. The results of Figure 10 (right side) allow to conclude that the MCMT filtering is slightly under optimal in terms of gain in $L_{e q}$ although, it remains very efficient. 

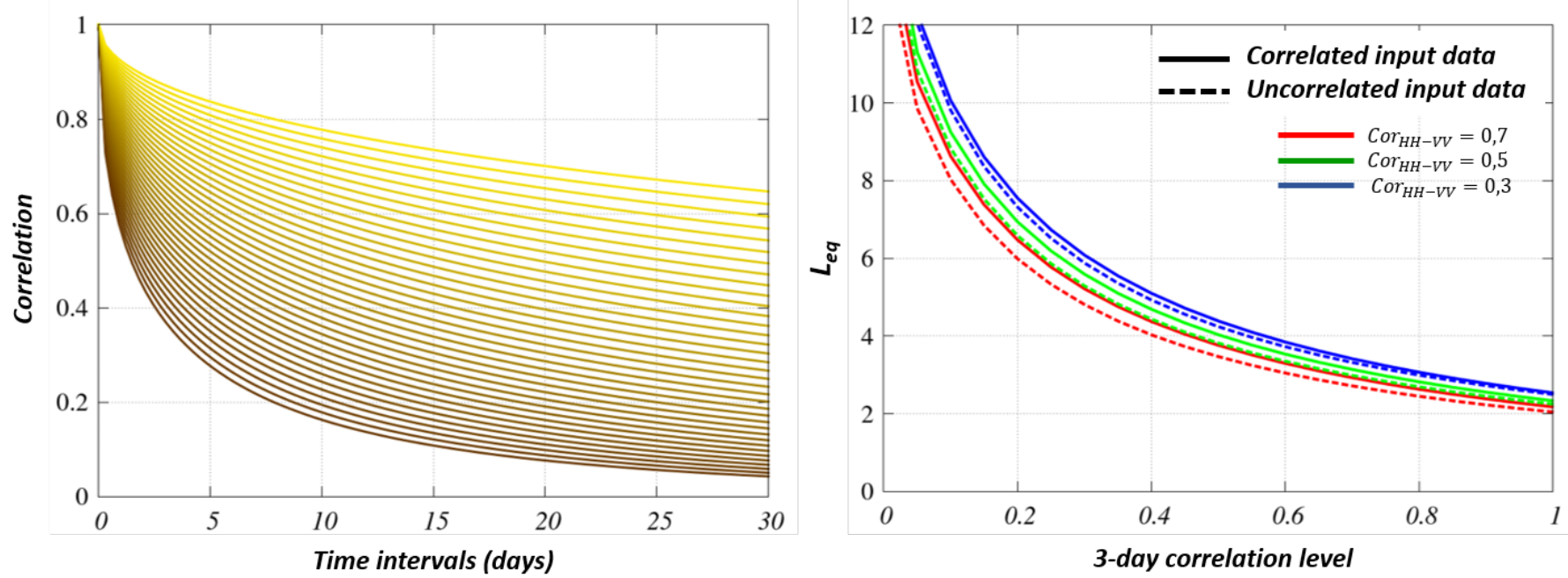

Figure 10. On the left: semi-empirical model of the repeat-pass correlation between the duration given along the $x$-axis, with upper and lower bounds corresponding to 3-day correlations of 0.87 and 0.37 respectively. On the right: theoretical $L_{e q}$ resulting from the filtering of 7 Biomass like polarimetric acquisitions as a function of the 3-day temporal correlation which determines the other temporal baseline (up to 18 days) through the semi-empirical decorrelation model (on the left). The various colors are for different stationary correlation between $\mathrm{HH}$ and $\mathrm{VV}$, ranging from 0.7 to 0.3 , and the dot and plain lines are for the optimal or non-optimal versions of the MCMT filter respectively.

\section{Conclusions and Further Prospects}

Through the implementation of an innovative speckle filter adapted to temporal sequences of polarimetric SAR SLC images, this paper puts forward a new performance indicator in order to assess the filtering efficiency, particularly relevant at P-band in the case where dense vegetation would jeopardize the microwave penetration through the underlying ground.

In agreement with the qualitative improvement of texture throughout the filtered images, this indicator enables to quantify the filter benefits, while the standard metric based on the Equivalent Number of Looks (ENL) fails to capture these changes. The saturation of the ENL has not been fully understood yet, but the most likely explanation for us lies in the intrinsic spatial correlation due to the forest structure.

Being based on the correlation between the polarimetric orientation angle and the azimuthal component of the ground slope estimated from an independent DEM, it is also worth noting that this indicator requires fully polarimetric SAR data and a DEM adapted to the microwave penetration. In our case with P-band SAR data, the DEM provided by the SRTM product at $30 \mathrm{~m}$ provides a good proxy of the Digital Terrain Model (DTM).

Together with speckle reduction, the implementation of such a filter contribute to preserve the spatial resolution, as well as the temporal resolution since the individual mean of each sample of the temporal sequence is maintained. These two features are particularly relevant in the frame of the BIOMASS dedicated to the temporal survey of the above ground biomass distribution at global scale, in which the spatial range resolution is limited by the $6 \mathrm{MHz}$ bandwidth allocated to civilian spaceborne applications. In addition, the preservation of the fully polarimetric covariance matrix is also very relevant, whether to implement backscattering coefficients like $t^{0}$ in order to optimize the sensitivity to forest biomass, or to exploit the polarimetric channels in order to cope with perturbing effects due to temporal variability.

This work also highlights the relevance of using the longest possible time series in order to benefit from uncorrelated images, given that the filter optimization to correlated images has shown a limited interest. Further prospects of development will also be dedicated to the combined use of non-local filters, as well as the filter implementation to other datasets in order to better cope with scenarios of correlated images. Beyond the 
direct objectives of BIOMASS, this work opens new perspectives of terrain slope estimation and DTM reconstruction from PolSAR data, considering that ascending and descending orbit are truly promising to cope with the method limitation related to the estimation of azimuthal components only.

Author Contributions: Since this study is part of her PhD work, C.G. is the first and corresponding author of the paper. L.F.-F., T.K. and L.V. supervised filter design and implementation, as well as result analysis and interpretation. As PhD advisors, S.D. and L.P. have contributed to coordinate this work and its writting. All authors have read and agreed to the published version of the manuscript.

Funding: This work was funded by the French government in partnership between Capgemini and CNES during a PhD work, and also benefited from CNES support through the TOSCA research programme.

Institutional Review Board Statement: Not applicable.

Informed Consent Statement: Not applicable.

Data Availability Statement: Samples of data are available from the authors.

Acknowledgments: The authors would like to thank Clement Albinet for his key role and support in the preliminary version of the future ESA BIOMASS processing platform facility. The authors are also grateful to Milena Planells for her kind support during the writing of this article.

Conflicts of Interest: The authors declare no conflict of interest.

\begin{tabular}{|c|c|}
\hline \multicolumn{2}{|c|}{ Abbreviations } \\
\hline \multicolumn{2}{|c|}{ The following abbreviations are used in this $\mathrm{m}$} \\
\hline 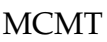 & \\
\hline $\mathrm{OA}$ & Pola \\
\hline AGB & round Biomass \\
\hline DEM & Digital Elevation Model \\
\hline SRTM & Shuttle Radar Topography Missio \\
\hline ENL & Equivalent Number of Looks \\
\hline RMSE & Root Mean Squared Error \\
\hline SLC & Single Look Complex \\
\hline
\end{tabular}

\section{References}

1. D'Alessandro, M.M.; Tebaldini, S.; Quegan, S.; Soja, M.; Ulander, L.M.H. Interferometric Ground Notching of SAR Images for Estimating Forest Above Ground Biomass. In Proceedings of the IGARSS 2018-2018 IEEE International Geoscience and Remote Sensing Symposium, Valencia, Spain, 22-27 July 2018; pp. 8798-8800.

2. Global Climat Observing System (GCOS). Systematic Observation Requirements for sAtellite-Based Products for Climate, Supplemental Details to the Satellite-Based Component of the Implemention Plan for the Global Observing System for Climate in Support of the UNFCCC; WMO/TD No 1338; 2006. Available online: https:/ / climate.esa.int/sites/default/files/gcos-154.pdf (accessed on 20 September 2020).

3. Hamadi, A.; Villard, L.; Borderies, P.; Albinet, C.; Koleck, T.; Le Toan, T. Comparative Analysis of Temporal Decorrelation at P-Band and Low L-Band Frequencies Using a Tower-Based Scatterometer Over a Tropical Forest. IEEE Geosci. Remote Sens. Lett. 2017, 14, 1918-1922. [CrossRef]

4. Agency, E.S. Biomass Coreh2o Premier: Report for Mission Selection. An Earth Explorer to Observe Forest Biomass; European Space Agency (ESA): Paris, France, 2012.

5. Lee, J.-S.; Schuler, D.L.; Ainsworth, T.L. Polarimetric SAR data compensation for terrain azimuth slope variation. IEEE Trans. Geosci. Remote Sens. 2000, 38, 2153-2163.

6. Lee, J.-S.; Jurkevich, L.; Dewaele, P.; Wambacq, P.; Oosterlinck, A. Speckle filtering of synthetic aperture radar images: A review. Remote Sens. Rev. 1994, 8, 313-340. [CrossRef]

7. Dubois-Fernandez, P.; Le Toan, T.; Chave, J.; Blanc, J.; Daniel, S.; Oriot, H.; Arnaubec, A.; Rejou-Mechain, M.; Villard, L.; Lasne, Y.; et al. Technical Assistance for the Development of Airbone SAR and Geophysical Measurments during the TropiSAR 2009 Experiment (Report); European Space Agency: Paris, France, 2011. [CrossRef]

8. Hamadi, A.; Borderies, P.; Albinet, C.; Koleck, T.; Villard, L.; Minh, D.H.T.; Le Toan, T.; Burban, B. Temporal coherence of tropical forests at P-band: Dry and rainy seasons. IEEE Geosci. Remote Sens. Lett. 2015, 12, 557-561. [CrossRef] 
9. Villard, L.; Le Toan, T. Relating P-Band SAR Intensity to Biomass for Tropical Dense Forest in Hilly Terrain : $\gamma^{0}$ et $t^{0}$ ? IEEE J. Sel. Top. Appl. Earth Obs. Remote Sens. 2015, 8, 214-223. [CrossRef]

10. Simonetto, L.P.E. Effect of scale on the correlation between topography and canopy elevations in an airborne InSAR product over Amazonia. Procedia Technol. 2014, 16, 180-185. [CrossRef]

11. Labriere, N.; Tao, S.; Chave, J.; Scipal, K.; Le Toan, T.; Abernethy, K.; Alonso, A.; Barbier, N.; Bissiengou, P.; Casal, T.; et al. In Situ Reference Datasets from the TropiSAR and AfriSAR Campaigns in Support of Upcoming Spaceborne Biomass Missions. IEEE J. Sel. Top. Appl. Earth Obs. Remote. Sens. 2018, 11, 3617-3627. [CrossRef]

12. Chave, J.; Réjou-Méchain, M.; Búrquez, A.; Chidumayo, E.; Colgan, M.S.; Delitti, W.B.; Duque, A.; Eid, T.; Fearnside, P.M.; Goodman, R.C.; et al. Improved allometric models to estimate the aboveground biomass of tropical trees. Glob. Chang. Biol. 2014, 20, 3177-3190. [CrossRef] [PubMed]

13. Yu, S.Q.J. Filtering of Multichannel SAR Images. IEEE Trans. Geosci. Remote Sens. 2001, 39, $2373-2379$.

14. Lee, J.S.; Grunes, M.R.; De Grandi, G. Polarimetric SAR speckle filtering and its impact on classification. In Proceedings of the IGARSS'97, Singapore, 3-8 August 1997; pp. 1038-1040.

15. Goodman, J. Some fundamental properties of speckle. J. Opt. Soc. Am. 1976, 66, 1145-1150. [CrossRef]

16. Le Toan, T.; Floury, N. On the Retrieval of Forest Biomass from SAR Data. Int. J. Remote Sens. 1994. [CrossRef]

17. Garestier, F.; Le Toan, T. Estimation of a forest backscatter profile at P-band using Single Baseline Pol-InSAR. IEEE Trans. Geosci. Remote Sens. 2010, 48, 3340-3348. [CrossRef]

18. Casal, T.; Hajnsek, I.; Pardini, M.; Jager, M.; Horn, R.; Kim, J.S.; Papathanassiou, K.; Dubois-Fernandez, P.; Dupuis, X.; Wasik, V.; et al. Technical Assistance for the Development of Airborne SAR and Geophysical Measurements during the Afrisar ExperimentDeliverable DD-4_Final Report; European Space Agency (ESA): Paris, France, 2016. [CrossRef] 